\title{
Current status of the R-Matrix code SAMMY, with emphasis on the relationship to ENDF formats
}

\author{
N.M. Larson ${ }^{\text {a }}$, L.C. Leal, R.O. Sayer, H. Derrien, D. Wiarda, and G. Arbanas
}

Oak Ridge National Laboratory, P.O. Box 2008, Oak Ridge, TN 37831-6171, USA

\begin{abstract}
During the last decade, advances in evaluations of neutron-induced cross sections in the resolved resonance region have surpassed the ability of the Evaluated Nuclear Data Files (ENDF) to make use of those evaluations. At the higher energies used in new evaluations, effects that in the past were deemed to be negligible are now important. There is also a great demand for including uncertainty information in ENDF files. In this paper, we describe recently approved ENDF formats to accommodate new evaluations and their implementation in SAMMY. Other new features of the SAMMY code, available in the 2006 Revision 7 release, are also described.
\end{abstract}

\section{Introduction}

The computer code SAMMY [1] is widely used for evaluation of neutron cross-section data in the resonance region. As a final step in the evaluation process, R-matrix parameters are written into ENDF File 2 and the associated resonance parameter covariance matrices are written into ENDF File 32. These files are then submitted to the various governing bodies for eventual inclusion in the evaluated nuclear data files.

In recent years, not all evaluations could be made to fit the confines of the ENDF formats. Modified or new formats have therefore been created to accommodate these evaluations. In this paper, we first discuss the new ENDF formats and then describe other new features of the SAMMY code.

\section{Creation of ENDF file 2}

As both experimental and theoretical techniques have improved, the resolved resonance region has been pushed to higher energies than previously possible. At those higher energies, previously unused aspects of R-matrix theory now appear more often. Inelastic channels, proton channels, alpha channels, or other reaction channels may be encountered. Larger values of orbital angular momentum $l$ are needed, requiring larger values of total spin $J$. Spin admixture (e.g., $l=0$ and $l=2$ ) may occur, even for initial states (elastic channels). Spin admixture may also occur for channel spin $s$ (defined by the vector sum of $I$ and $i$, where $I$ is the spin of the target nuclide and $i=1 / 2$ is the spin of the neutron). Because $J$ is the vector sum of $l$ and $s$, it is possible for many channels to contribute to some spin groups. (A "spin group" is defined by $J$ and parity, the only conserved quantities.)

The ENDF format used for most Reich-Moore evaluations is defined by the parameter LRF $=3$ and is denoted the "Reich-Moore format" [2]. This format is unable to accommodate the effects described above, because it uses only a highly restricted form of the actual Reich-Moore formulation [3]. With LRF $=3$, only one incident channel and at most two fission channels are permitted. No threshold channels of any

\footnotetext{
a Presenting author, e-mail: LarsonNM@ornl .gov
}

kind are allowed, and no orbital angular momentum or channel spin admixtures are permitted. Some information (the value of $J$ ) is repeated for every resonance, while many other quantities are completely undefined (parity; charge; and sometimes, channel spin). At least one implicit assumption is made (the existence of a second "spin group" with the same $l, J$, and $\pi$ and the opposite channel spin $s$ ). In the past, these undefined quantities and implicit assumptions have caused misunderstanding between the intended evaluated cross section and the cross section as reproduced by the processor codes.

One example of such confusion is shown in figure 1, in which two possible interpretations of the ${ }^{27} \mathrm{Al}$ total cross section is shown. Two resonances with the same $J^{\pi}$ and the same $l$ occur in close proximity to one another. In the original evaluation, these were assigned to different channel spins and therefore did not interfere with each other. In the original $\mathrm{LRF}=3$ format, there was no mechanism for assigning different channel spins; hence, processor codes interpreted this as destructive interference between two resonances of the same spin group, as indicated by the dotted curve. The measured data, however, were well represented by the solid curve in the figure.

In 1999 a remedy for this situation was adopted. The LRF $=3$ format included an unsigned value of the spin $J$ for each resonance; R.Q. Wright [4] recognized that the sign on that quantity could be used to define the channel spin $s$. The convention is as follows: Let AJ represent the value printed in the ENDF File 2. When AJ is negative, then $s=I-1 / 2$. When AJ is positive, then $s=I+1 / 2$ (unless this is an older file for which the value of $s$ is irrelevant). The total angular momentum $J$ is the absolute value of AJ.

The cross-section values calculated using this modified format are identical to the original evaluated values, shown in the solid curve in figure 1.

The 1999 patch was helpful, but did not address all the limitations inherent in the LRF $=3$ format. A new ENDF format was therefore created; the format is denoted by LRF = 7 and labeled "R-Matrix Limited". ("Limited" suggests that there are some possible R-matrix features that are not included here; a non-hard-sphere phase shift is one example.) This format was approved by the Cross Section Evaluation Working Group (CSEWG) in 2004. 


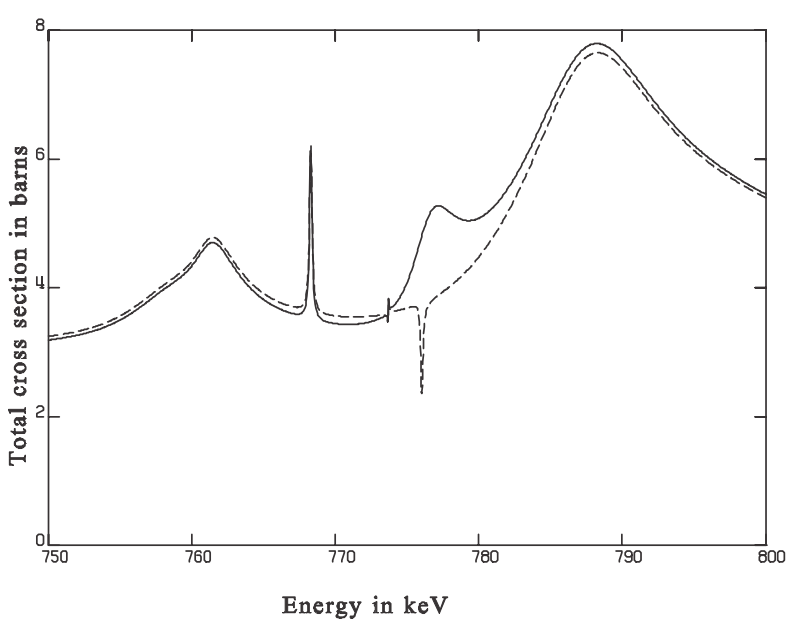

Fig. 1. Incorrect ${ }^{27} \mathrm{Al}$ cross-section values produced in past years from incomplete ENDF LRF $=3$ format (dotted curve). The correct cross-section values, produced from the modified ENDF LRF $=3$ format, are shown by the solid curve.

The LRF $=7$ format is fully compatible with the Rmatrix formulation used in the SAMMY analysis code [1]. Particle pairs are defined by specifying quantum numbers (spin, parity, charge, and mass) for the two particles and/or by giving the type of interaction (capture, fission, etc.). For a given spin group (a given $J^{\pi}$ ), each available channel is defined by specifying the particle pair, the orbital angular momentum, and the channel spin. If resonances exist for the spin group, resonance parameters are given.

Figure 2 shows an example of the LRF $=7$ format for ${ }^{16} \mathrm{O}$, for which there is a low-lying alpha channel. Other examples can be found in the SAMMY test cases (e.g., test case number 126; see section XII of ref. [1]) and in ref. [5].

The LRF $=7$ format can be both written and read by the SAMMY code. Use of this format has also been implemented within several processor codes, such as AMPX [6] and NJOY [7]. Anyone wishing to incorporate this expanded R-matrix capability into his or her own code (or to compare it with an independent implementation) may contact N.M. Larson to request a copy of SAMRML.

\section{Creation of ENDF file 32}

A second recent development in the evaluation process is the enhanced use of uncertainty information. A concerted effort is currently under way to provide reasonable and reliable estimates of uncertainty information for all ENDF files.

Techniques have been incorporated into SAMMY for userfriendly, efficient treatment of measurement-related uncertainties (both statistical and systematic). The output resonance parameter covariance matrix (RPCM) is expected to reflect those uncertainties. It is also possible to include measured thermal data and resonance integral information, which can have a significant impact on the evaluated differential uncertainties.

For older evaluations lacking File 32 covariance information, schemes have been developed for retroactively
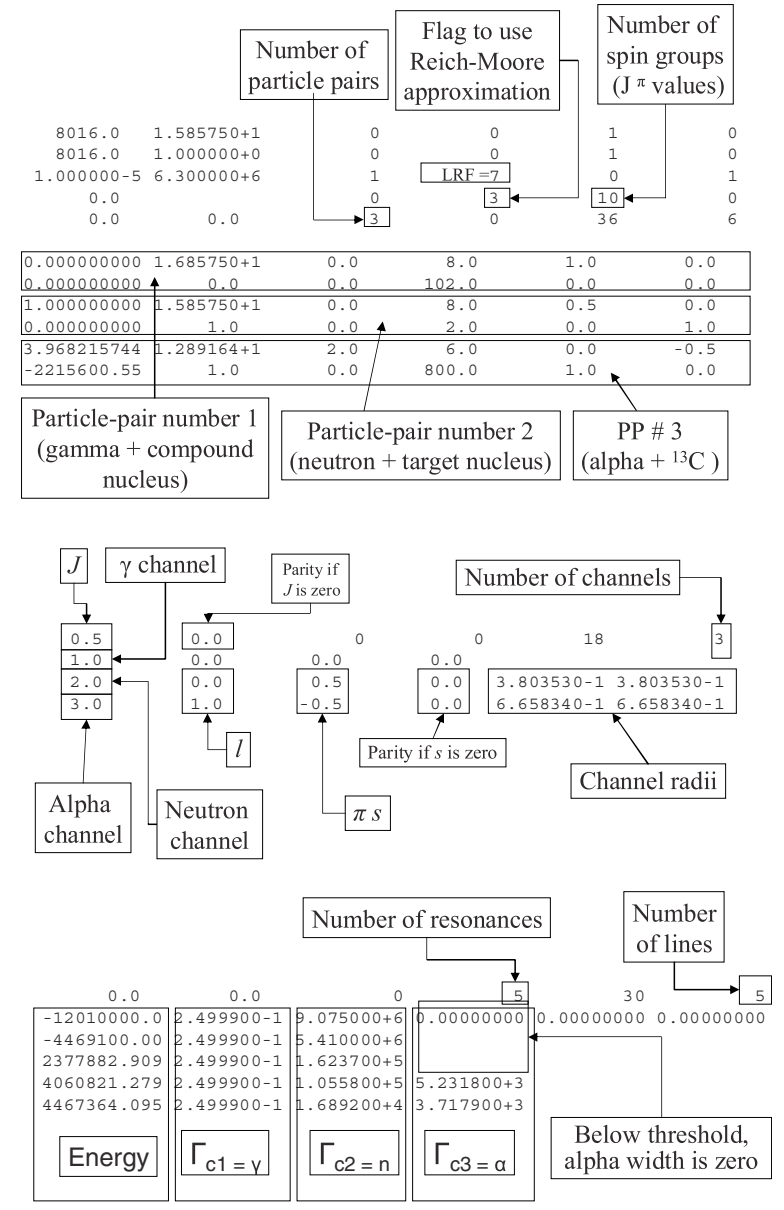

Fig. 2. Example fo ENDF LRF $=7$ format for the case of $\mathrm{O}^{16}$ including an alpha channel.

generating approximate RPCMs. Additional details about these techniques can be found in the SAMMY users' manual [1] and in other papers [8].

When evaluations are prepared for submission to ENDF, the RPCM is written into File 32. For most nuclides, the socalled "short-range component" of the File 32 LCOMP $=1$ format can be used to communicate the entire RPCM. For nuclides with many resonance parameters (e.g., ${ }^{235} \mathrm{U}$ ), the large size of the covariance matrix would prohibit use of the complete RPCM in the ENDF file under current Data Bank policy. This has led to the development of a new "compact" format, capable of communicating the major features of the RPCM in an abbreviated space.

The compact format, defined by LCOMP = 2 and approved by CSEWG in 2004, involves conversion from covariance matrix to uncertainties plus correlation matrix. Small correlation coefficients (below $\sim 2 \%$ ) are assumed to be negligible; larger values are rounded to the nearest percent and then stored as signed two-digit integers.

The LCOMP $=2$ format, as well as the original LCOMP $=$ 1 format, has been implemented in the PUFF-IV [9] module of the AMPX [6] code. PUFF-IV can produce covariance matrices for multigroup cross sections over the entire ENDF energy range. 


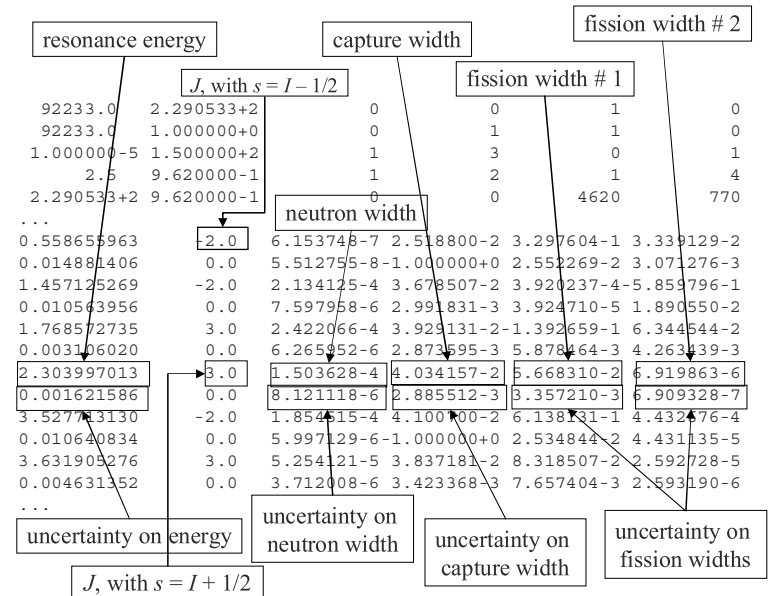

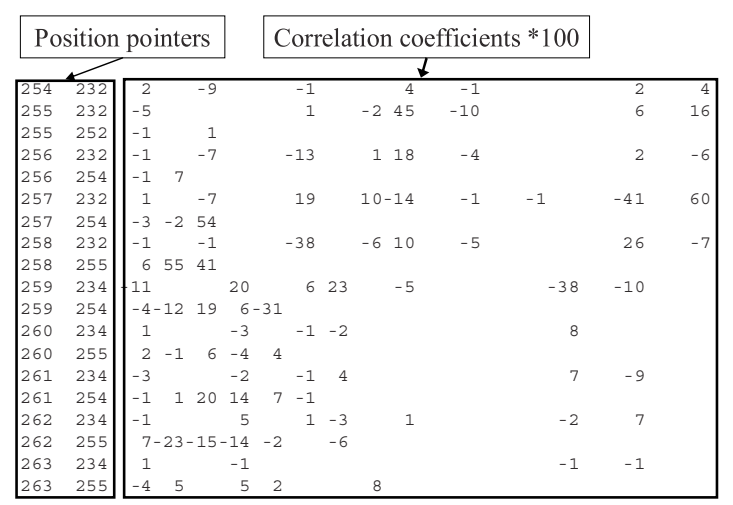

Fig. 3. Small portion of ENDF File 32 with LCOMP $=2$ for the case of ${ }^{233} \mathrm{U}$

A small portion of an ENDF File 32 with LCOMP $=2$ for ${ }^{233} \mathrm{U}$ is shown in figure 3 .

When the compact format was first proposed, it was known to be a relatively crude approximation. Preliminary tests had shown that it could reduce the length of the ENDF file significantly and that the reconstructed covariance matrix was comparable to the original. Unfortunately, on the larger covariance matrices for which this format was designed, the approximation seems to work less well; multigroup covariance matrices constructed from the compact RPCM occasionally show unacceptable behavior (have negative diagonal elements, in the worst-case scenario). One stop-gap measure was approved by CSEWG at the 2006 meeting, that being the extension to $3,4,5$, or 6 digits for storing the correlation matrix elements.

A better solution is clearly needed. Within the confines of current ENDF philosophy, the one promising alternative is to use the largest eigenvalues of the correlation matrix; this option will be studied in more detail, and a format proposal will likely be developed.

A more radical departure from the current ENDF philosophy may ultimately be required. All ENDF 11-digit ASCII covariance formats have the same potential problem: conversion to ASCII involves dropping significant digits on some of the numbers, which can easily lead to nonpositive definite covariance matrices. Higher precision format options should therefore be considered.

\section{Other new features in SAMMY}

Much of the recent development in the SAMMY code is related specifically to ENDF formats.

SAMMY now has capabilities for reading and writing File 2 (resonance parameters) in four different formats: LRF = 1 (single-level Breit-Wigner); LRF $=2$ (multilevel BreitWigner, which is actually single level except for the elastic cross section); LRF = 3 (Reich-Moore); and LRF = 7 (RMatrix Limited). (SAMMY users are, however, urged to use the Breit-Wigner formats only for reading old ENDF files and never for creating new files. There is no need to perform new evaluations using the sometimes unphysical Breit-Wigner approximations, because no penalty is associated with using the more rigorous Reich-Moore approximation.)

Likewise, SAMMY is capable of reading and writing File 32 in most of the existing formats, though not with all possible options. The recommended format is LCOMP $=1$ with no "long-range component"; that is, the entire resonance parameter covariance matrix should be written as one "shortrange" section that covers the entire resolved resonance energy region. For those few instances in which this gives a prohibitively large file, LCOMP = 2 may be a viable alternative.

There are other new SAMMY features in addition to those related to ENDF. Features in place prior to October 2006 are listed in the introduction to the SAMMY users' manual [1] and described in detail within that document; most will not be discussed here. Two that should be noted are the implicit data covariance (IDC) method and the propagated uncertainty parameter (PUP) technique, both of which allow easier, more-efficient, and more-rigorous treatment of measurement uncertainties, thus leading to the creation of more-realistic resonance parameter covariance matrices.

Features added subsequent to the 2006 release of the manual include

- energy-dependent $v$ (for $\eta$ calculations),

- a resolution function option for a Gaussian width that is constant in energy (useful for incident charged particles),

- an option to input the resonance widths as reduced-width amplitudes rather than partial widths (useful when resonances lie very near threshold),

- the ability to include both the Reich-Moore eliminated gamma channel and any individually specified gamma channels when calculating capture cross sections.

In addition to the new features being added to the code, documentation is being completed for the multiple-scattering corrections for capture or fission yields. One added benefit of the documentation process is improvement in the calculation of the partial derivatives for these important corrections.

\section{Conclusions}

Modern evaluation techniques have necessitated improvements in existing ENDF formats or creation of new formats for resonance parameters and the associated covariance matrix. Several such formats have been created, implemented, and 
tested by members of the Nuclear Data Group at Oak Ridge National Laboratory, and finally approved by CSEWG as official ENDF formats.

Not all recent work on the SAMMY code has been concerned with ENDF formats. Other new options continue to be developed; these were summarized in section 4 of this paper.

This work was sponsored by the US Department of Energy Nuclear Criticality Safety Program (NCSP), under contract DEAC0500OR22725 with UT-Battelle, LLC.

\section{References}

1. N.M. Larson, Updated Users' Guide for SAMMY: Multilevel R-Matrix Fits to Neutron Data Using Bayes' Equations, ENDF364/R1 and ORNL/TM-9179/R7, Oak Ridge National Laboratory, Oak Ridge, TN (September 2006). Available at http://www.ornl.gov/sci/nuclear_science_technology/nuclear_data sammy/.

2. ENDF-102, Data Formats and Procedures for the Evaluated Nuclear Data File, ENDF-6, written by the Members of the Cross Section Evaluation Working Group, edited by V. McLane,
C.L. Dunford, P.F. Rose, BNL-NCS-4495, Brookhaven National Laboratory, April 2001. Updated BNL-NCS-4495/04-Rev, edited by M. Herman, June 2005.

3. C.W. Reich, M.S. Moore, Phys. Rev. 111, 929 (1958).

4. R.Q. Wright (1999) (private communication).

5. N.M. Larson, SAMMY User Guidance for ENDF Formats, ENDF367 and ORNL/TM-2007/23, Oak Ridge National Laboratory, Oak Ridge, TN (March 2007).

6. M.E. Dunn, N.M. Greene, AMPX-2000: A Cross-Section Processing System for Generating Nuclear Data for Criticality Safety Applications, Trans. Am. Nucl. Soc. 86 (2002), pp. 118-119.

7. R.E. MacFarlane, D.W. Muir, R.M. Boicourt, The NJOY Nuclear Data Processing System, Vol. II: The NJOY, RECONR, BROADR, HEATR, and THERMR Modules, LA-9303-M, Vol. II (ENDF-324) (May 1982). Also R.E. MacFarlane, Introducing NJOY 89, LA-UR 89-2057, June 1989.

8. N.M. Larson, Use of Covariance Matrices in SAMMY, invited paper at the Workshop on Nuclear Data Evaluation for Reactor application (WONDER 2006) held at the Château de Cadarache, October 9-11, 2006.

9. D. Wiarda, M.E. Dunn, PUFF-IV: A Code for Processing ENDF Uncertainty Data into Multigroup Covariance Matrices, ORNL/TM-2006/147, Oak Ridge National Laboratory, Oak Ridge, TN (October 2006). 